\title{
Cytochemical and Immunocytochemical Localization of Oxidative and Nitrosative Stress in Pulmonary Hypertension in Lungs of Broiler Chickens, Gallus domesticus
}

\author{
Jaime Bautista-Ortega ${ }^{*}$, C. A. Ruiz-Feria ${ }^{*}$, and E. Ann Ellis ${ }^{* *}$ \\ *Department of Poultry Science and ${ }^{* *}$ Microscopy and Imaging Center, BSBW 119/MS 2257, Texas \\ A\&M University, College Station, TX 77843
}

Pulmonary hypertension and ascites affects young, rapidly growing broilers under conditions of hypoxia. Hypoxic pulmonary hypertension results in chronic right ventricular heart failure as well as cardiovascular stress in the lungs. In addition, the rapid weight gain of broilers has been suggested as a model for cardiovascular complications associated with obesity. Oxidative and nitrosative stresses have been implicated in the pathology of these vascular complications [1]. Elevated levels of nitric oxide (NO) and superoxide can uncouple constitutive endothelial nitric oxide synthase and reduce availability of NO. NADH oxidase has been shown to be a source of the free radical superoxide $\left(\mathrm{O}_{2}{ }^{-}\right)$in many cardiovascular disease processes and peroxynitrite $\left(\mathrm{ONOO}^{-}\right)$ has been documented in reduced availability of nitric oxide (NO) [2]. Initial studies were done to document the presence of NADH oxidase, a biomarker for $\mathrm{O}_{2}{ }^{-}$, and nitrotyrosine, a biomarker for $\mathrm{ONOO}^{-}$and reduced availability of NO.

Day old broilers chicks were raised for four weeks in hypobaric chambers which simulated the hypoxia at 10,000 ft above sea level. Control broilers were raised under normoxic conditions. All broilers received food and water ad libitum. At the end of the experiment all broilers were euthanized and lungs were fixed by perfusion through the heart with $4 \%$ (vol/vol) paraformaldehyde and $1 \%$ (vol/vol) glutaraldehyde in $0.1 \mathrm{M}$ HEPES buffer, $\mathrm{pH}$ 7.4. Lungs were processed for cytochemical localization of NADH oxidase and nitrotyrosine localization was done with colloidal gold based immunocytochemistry [3]. Controls for specificity of the NADH oxidase reaction consisted of omission of the substrate and use of a specific inhibitor, diphenyleneiodonium. Some samples were processed for scanning reflectance confocal miocroscopy of NADH oxidase localization and quantitation of the reaction product, cerium perhydroxide[4].

NADH oxidase localized in the vascular endothelial cells (Fig. 1) and the parabronchial epithelial cells of normoxic and hypoxic lungs (Fig. 2). Semiquantitative studies using reflectance intensity in confocal microscopy of cerium perhydroxide showed a 1.3 fold increase of NADH oxidase in hypoxic birds over normoxic birds (Table 1). There was extensive localization of nitrotyrosine (Fig. 3 ), the biomarker for $\mathrm{ONOO}^{-}$, and there was a 1.6 fold increase of nitrotyrosine in lungs of hypoxic birds as compared to normoxic birds (Table 1).

The broiler has been bred to convert feed to muscle mass at a rapid rate. However, at the same time these birds develop some of the same cardiovascular pathologies as obese patients. The pulmonary hypertension that develops in broilers as a result of hypoxia makes this a good model for studies of pulmonary hypertension. These preliminary studies document a role for oxidative and nitrosative stress in both vascular endothelium and parabronchial epithelium in the lungs of this broiler model of pulmonary hypertension. Increased levels of NADH oxidase and nitrotyrosine indicate reduced availability of NO in the lungs of hypoxic broilers with pulmonary hypertension. 
References

[1] W. G. Bottje and R. F. Wideman, Poultry and Avian Rev. 6 (1995) 211.

[2] H. Li et al., Nitric Oxide 7 (2002) 149.

[3] E. A. Ellis et al., Nitric Oxide 6 (2002) 304.

[4] J . M. Robinson and B. E. Batten, J. Histochem. Cytochem. 38 (1990) 315.

[5] This work was supported by Hatch Project H70280.

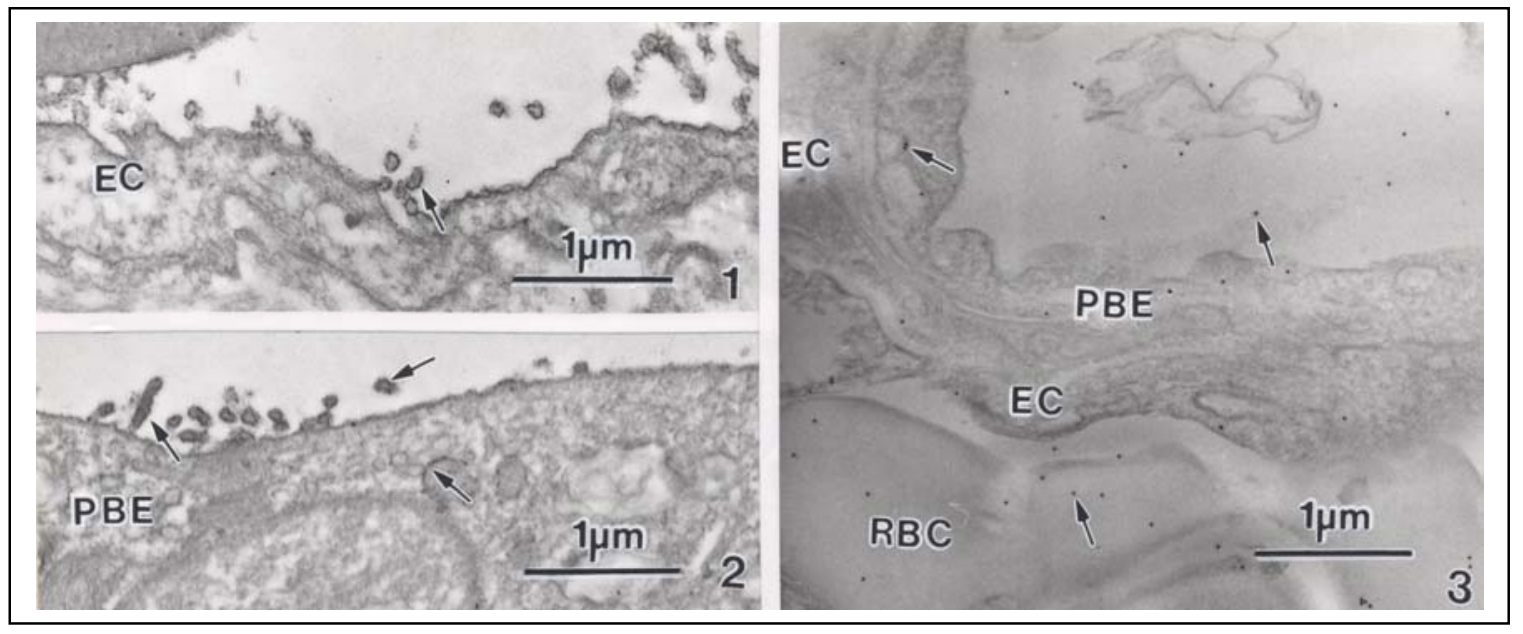

Fig.1 Cytochemical localization of NADH oxidase(arrows) in lung vascular endothelial cell (EC). Fig. 2 Cytochemical localization of NADH oxidase(arrows) in lung parabronchial epithelium (PBE). Fig. 3 Immunocytochemical localization of nitrotyrosine with $12 \mathrm{~nm}$ colloidal gold (arrows) in lung vascular endothelium(EC) and parabronchial epithelium (PBE). Red blood cells (RBC).

\begin{tabular}{|l|c|c|}
\hline BROILER TREATMENT & $\begin{array}{c}\text { NA DH OXIDASE } \\
\text { (mean reflectance units } \pm \\
\text { SEM) }\end{array}$ & $\begin{array}{c}\text { NITROTYROSINE } \\
\text { (mean colloidal gold particles/ } \\
\mathbf{1 0 0}^{\mathbf{2}} \pm \mathbf{\text { SEM}}\end{array}$ \\
\hline Hypoxic $(\mathrm{n}=6)$ & $405 \pm 15.6$ & $67 \pm 10$ \\
\hline Normoxic $(\mathrm{n}=3)$ & $326.3 \pm 22.2$ & $43 \pm 10$ \\
\hline
\end{tabular}

Table 1 Quantitation of NADH oxidase and nitrotyrosine in hypoxic and normoxic broilers. 